\title{
Reflections and Calculations on a Prey-Predator-Patch Problem
}

\author{
O. Diekmann \\ Centre for Mathematics and Computer Science \\ Kruislaan 413, 1098 SJ Amsterdam, the Netherlands \\ \& \\ Institute of Theoretical Biology, University of Leiden \\ Groenhovenstraat 5, 2311 BT Leiden, the Netherlands \\ J.A.J. Metz \\ Institute of Theoretical Biology, University of Leiden \\ Groenhovenstraat 5, 2311 BT Leiden, the Netherlands

\section{M.W. Sabelis ${ }^{*}$} \\ Department of Population Biology, University of Leiden \\ P.O. Box 9516, 2300 RA Leiden, the Netherlands
}

\begin{abstract}
This paper is concerned with models for the interaction of plants, herbivores and their predators. We concentrate on situations in which local colonies of herbivores either over-exploit their host plant or are driven to extinction by predators. Starting from a complicated structured model, in which the local prey and predator density within patches is taken into account, we use time scale arguments to derive a three dimensional system of ordinary differential equations. The simplified system is analysed and the existence of multiple stable steady states is demonstrated.
\end{abstract}

1980 Mathematics Subject Classification: $92 \mathrm{~A} 15$

Key Words \& Phrases: physiologically structured population models, predator-preyplant interaction, patch structure, model simplification, time scale arguments, qualitative analysis, multiple stable steady states

\section{INTRODUCTION}

This paper reports some recent work on a collection of mathematical models for the interaction of phytophages and their natural enemies in an ensemble of local patches of host plants. The key idea is to consider a local colony as an "individual" characterized by the number of prey $x$, the number of predators $y$ and, possibly, some index for the available food for the prey such as host plant leaf area or biomass. Once the dynamics at the "individual" level are specified one can employ a general methodology (essentially just correct bookkeeping; see Metz \& Diekmann, 1986) to derive a "population" model.

The ideal then is to understand the global dynamical behaviour and in particular

* Present address: Department of Pure and Applied Ecology, University of Amsterdam, Kruislaan 302, 1098 SM Amsterdam, the Netherlands. 
how this behaviour is affected by the various ingredients of the (sub)model(s). To attain this ideal for a nonlinear infinite dimensional dynamical system involving many parameters is a next to impossible task. Therefore we have to have recourse to simplifications.

A true understanding of natural phenomena quite often requires a whole spectrum of supplementary models rather than one particular model. We advocate the use of structured models to fill the gap between realistic but complicated simulation models on the one end and qualitative caricatures in terms of ordinary or functional differential equations on the other extreme. The exercise of formulating explicitly a complicated structured model is useful in itself since foggy notions are clarified in the process and questions are identified. As a next step time scale arguments (quasi steady-state assumptions or neglect of delays) or special choices of model ingredients may be employed to derive analytically tractable simplifications. Thus one obtains a coherent network of models, and qualitative insights derived from the simplest elements may be used to give direction to numerical experiments on the more intricate elements and to guide the interpretation of the outcomes.

In a recent survey (Diekmann, Metz \& Sabelis, 1988) we have illustrated this approach to the modelling of predator-prey interactions in a patchy environment by means of several examples of possible simplifications and the biological conclusions derived from these. In the present more limited paper we concentrate on one rather drastic simplification resulting in a system of three ordinary differential equations which we shall analyse in some detail.

In section 2 we present the structured "master" model while section 3 is devoted to a time scale argument and the resulting simplification. Section 4 deals with the existence, multiplicity and stability of steady states of the three dimensional ode system and, finally, in section 5 the main conclusions are translated into biological terms.

\section{MODEL FORMULATION}

Consider a herbivorous prey population living scattered over many local patches. New prey colonies are founded by individuals emigrating from existing prey colonies and invading "empty" patches of host plants. Prey colonies come to an end when the host plants are locally over-exploited or when predator invasion has eventually resulted in complete extermination of the prey followed by dispersal of the predators.

Let $x$ denote the number of prey in a given patch. Consider a patch in which only prey are present. We assume that the process of prey colony growth is described by the ordinary differential equation $\frac{d x}{d t}=v(x)$ until the host plant is locally over-exploited or the colony is invaded by a predator. If we assume that all empty patches offer an identical prospect for the prey then the number of prey in a colony which crashes due to host plant over-exploitation is a constant, which we shall call $x_{\max }$ (so $x_{\max }$ is the exploitable energy of an empty patch expressed in prey equivalents).

Let $Q(t)$ denote the number of potentially invading predators around at time $t$. Assuming mass action kinetics we let the per colony rate at which prey colonies of size $x$ are invaded be given by $\eta(x) Q(t)$, where the vulnerability $\eta$ describes how 
attractive (or, conspicuous) a prey patch of size $x$ is.

Let $n_{0}(t)$ denote the number of suitable empty patches at time $t$ and let $P(t)$ denote the number of potential prey colonists around at time $t$, then, again assuming mass action kinetics, the rate at which new prey colonies are founded is given by $\zeta n_{0}(t) P(t)$, where $\zeta$ denotes a reaction constant.

To describe the "population" level we now introduce the density function $n(t, x)$ which is such that the number of patches at time $t$ with prey level between $x_{1}$ and $x_{2}$ is given by

$$
\int_{x_{1}}^{x_{2}} n(t, \xi) d \xi
$$

Straightforward bookkeeping arguments (Metz \& Diekmann, 1986, p. 15, 92-97, 101) then yield the balance laws

$$
\left\{\begin{array}{l}
\frac{\partial n}{\partial t}(t, x)+\frac{\partial}{\partial x}(v(x) n(t, x))=-\eta(x) Q(t) n(t, x), \quad 1<x<x_{\max }, \\
v(1) n(t, 1)=\zeta n_{0}(t) P(t) .
\end{array}\right.
$$

Any invaded prey patch becomes a (prey-) predator patch. To describe such patches we introduce the number of predators $y$ as another state variable. We assume that the local prey-predator interaction is described by the system of ordinary differential equations

$$
\frac{d x}{d t}=g(x, y) \quad, \quad \frac{d y}{d t}=h(x, y) .
$$

Let the density function $m(t, x, y)$ be such that at time $t$ the number of patches with prey level between $x_{1}$ and $x_{2}$ and predator level between $y_{1}$ and $y_{2}$ is given by

$$
\int_{x_{1}}^{x_{2}} \int_{y_{1}}^{y_{2}} m(t, x, y) d y d x
$$

then our assumptions entail the balance laws

$$
\left\{\begin{array}{l}
\frac{\partial}{\partial t} m(t, x, y)+\frac{\partial}{\partial x}(g(x, y) m(t, x, y))+\frac{\partial}{\partial y}(h(x, y) m(t, x, y))=0 \\
h(x, 1) m(t, x, 1)=\eta(x) Q(t) n(t, x)
\end{array}\right.
$$

A precise description of the domain in the $(x, y)$-plane in which the differential equation holds requires a submodel for host plant consumption by the prey in order to compute the "resource exhaustion boundary" (see Metz \& Diekmann, 1986, p.82 for the simplest possible example). Here we shall neglect this point since the limiting case we are going to consider is chosen such that it becomes irrelevant. The assumption that the predators drive the prey locally to extinction translates into the assumption that the orbits of the prey-predator interaction system connect the invasion boundary $y=1$ with the extermination boundary $x=0$.

Let $\mu$ and $\nu$ denote the death rates of, respectively, the prey and predator aireal plankton. In accordance with our previous assumptions we describe the dynamics 
of $P$ and $Q$ by (see Metz \& Diekmann, 1986, p. 98-99)

$$
\begin{aligned}
& \frac{d P}{d t}(t)=x_{\max } v\left(x_{\max }\right) n\left(t, x_{\max }\right)-\mu P(t) \\
& \frac{d Q}{d t}(t)=-\int_{1}^{y_{\max }} y g(o, y) m(t, o, y) d y-\nu Q(t)
\end{aligned}
$$

where we have ignored the possible increase of $P$ and $Q$ due to prey and predators dispersing from patches reaching the resource exhaustion boundary in the $(x, y)$ plane. Concerning the number of empty patches $n_{0}$ we shall assume that

$$
\frac{d n_{0}}{d t}=f\left(n_{0}\right)-\zeta n_{0} P
$$

where $f$ is, for example, the familiar logistic function

$$
f\left(n_{0}\right)=r n_{0}\left(1-\frac{n_{0}}{K}\right) \text {. }
$$

Provided with appropriate initial conditions the equations (2.1) - (2.5) yield a complete dynamical description of the system.

\section{INSTANTANEOUS hOST PLANT DESTRUCTION}

Suppose the prey exhaust their host plant very quickly compared with the time scale of dispersal, then the founding of a prey colony leads almost instantaneously to the production of new searching prey unless predator invasion precludes overexploitation in which case the yield consists of predators rather than prey. How do we translate this verbal description of a limiting case into a mathematical simplification of (2.1) - (2.5)?

Solving (2.1) and (2.2) by integration along characteristics (see e.g. Metz \& Diekmann, 1986, p. 68-69, 104-105) one can express $n(t, x)$ and $m(t, x, y)$ in past values of $n_{0}, P$ and $Q$. Substitution of these expressions into (2.3) - (2.5) then yields a closed system of three delay differential equations. In the limiting case this becomes a system of three ordinary differential equations which describes the system by following the number of empty patches as well as the prey and predator aireal plankton as a function of time, while the rise and annihilation of local colonies are reduced to point events.

To actually calculate the right-hand side of the differential equations we specialize by taking $v(x)=\alpha x, g(x, y)=\alpha x-\beta y$ and $h(x, y)=\gamma y$ (that is, we take exponential prey growth in the absence of predators and a constant functional and numerical response) and let $\alpha, \beta, \gamma \rightarrow \infty$. To let predator invasion still be appreciable we have to let $\eta \rightarrow \infty$ as well with $\alpha$ and $\eta$ of the same order. The interpretation suggests to take $\beta$ and $\gamma$ of the same order. In order to avoid the complication of patches reaching the resource exhaustion boundary in the $(x, y)$-plane we let $\frac{\alpha}{\gamma} \rightarrow 0$ or, in other words, we assume that the predators reproduce an order of magnitude faster than the prey. In Appendix II of Diekmann, Metz \& Sabelis (1988) it is shown that under these assumptions the limiting system of ode's is 


$$
\begin{aligned}
& \frac{d n_{0}}{d t}=f\left(n_{0}\right)-\zeta n_{0} P \\
& \frac{d P}{d t}=x_{\max } \zeta n_{0} P e^{-\omega Q}-\mu P \\
& \frac{d Q}{d t}=\zeta n_{0} P h(Q)-\nu Q
\end{aligned}
$$

where by definition

$$
\begin{aligned}
& \omega=\int_{1}^{x_{\max }} \frac{\eta(\sigma)}{\alpha \sigma} d \sigma \\
& h(Q)=\frac{\beta}{\gamma} \int_{1+\frac{\gamma}{\beta}}^{1+\frac{\gamma}{\beta} x_{\max }} y d\left[1-e^{-Q}\left\{\frac{\frac{\beta}{\gamma}(\omega-1)}{\alpha \sigma} d \sigma\right]\right.
\end{aligned}
$$

So the rate of production of prey aireal plankton equals the product of the yield factor $x_{\max }$, the rate of founding of new prey colonies $\zeta n_{0} P$ and a reduction factor $\exp (-\omega Q)$ to account for predator invasion. The function $h$ describes how the mean yield of predators per founded prey patch depends on the current predator aireal plankton $Q(t)$. (Note that the yield in predators depends on the size of the prey colony at the moment of invasion while the probability of invasion at some particular size depends on the vulnerability $\eta$ as well as on $Q$. The per capita yield $h(Q) / Q$ is monotone decreasing.)

Specializing still further we take $\eta(x)=\alpha \delta x$ which means that we assume that the probability of predator invasion is proportional to the prey colony size. Then

$$
\begin{aligned}
& \qquad \begin{array}{l}
\omega=\delta\left(x_{\max }-1\right) \\
\text { and } h(0)=\lim _{Q \downarrow 0} h(Q)=0 . \text { Here } \\
\left.\rho=\frac{x_{\max }-1}{x_{\max }+\beta / \gamma}+x_{\max }\right)\left(1-e^{-\omega Q}+\rho\left(\frac{1-e^{-\omega Q}}{\omega Q}-1\right)\right), Q>0
\end{array}
\end{aligned}
$$

The graph of $h$ is sketched in Figure 1. Note that $h$ decreases for large values of $Q$ ! One can prove analytically that $h$ has exactly one maximum for positive $Q$.

In the next section we shall analyse the system (3.1) with the empty patch production function $f$ given by

$$
f\left(n_{0}\right)=r n_{0}\left(1-\frac{n_{0}}{K}\right)
$$

The relation between $h$ and $Q$ for $\omega=1, \frac{\beta}{\gamma}+x_{\max }=1$ and various values of $\rho$ 
O. DIEKMANN ET AL.

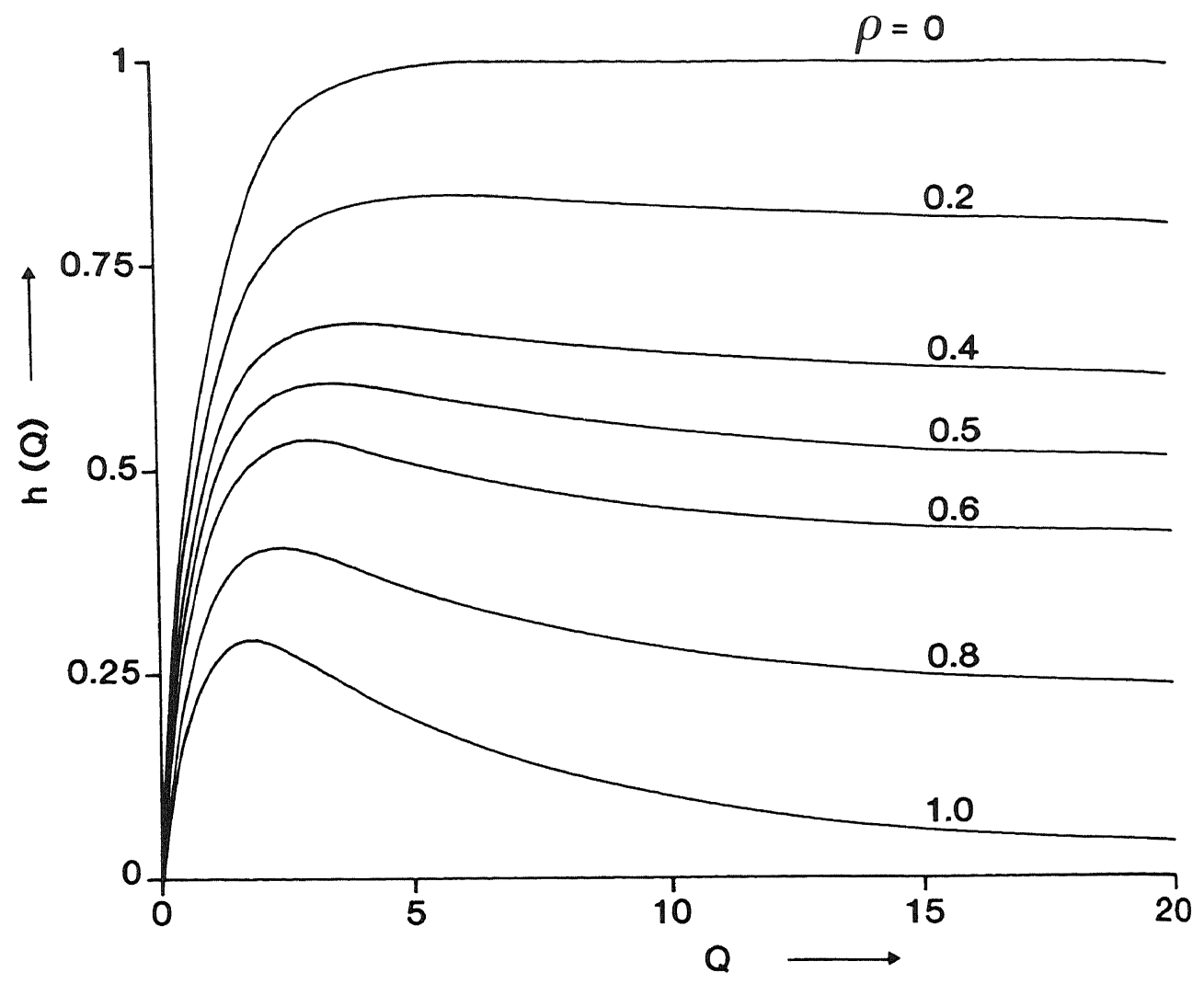

FIGURE 1

4. STABILITY AND BIFURCATION

We begin our analysis of (3.1) (with $\omega, h, \rho$ and $f$ given by (3.2) - (3.5)) by performing a scaling. Define

$$
\begin{aligned}
& \left\{\begin{array}{l}
u(t)=\frac{x_{\max } \zeta}{\mu} n_{0}\left(\frac{t}{\mu}\right) \\
v(t)=\frac{\zeta}{\mu} P\left(\frac{t}{\mu}\right) \\
w(t)=\omega Q\left(\frac{t}{\mu}\right)
\end{array}\right. \\
& \begin{cases}a=\frac{\mu \omega}{x_{\max } \zeta}\left(\frac{\beta}{\gamma}+x_{\max }\right) & b=\frac{r}{\mu} \\
c=\frac{x_{\max } \zeta K}{\mu} & d=\frac{\nu}{\mu}\end{cases}
\end{aligned}
$$


then the system (3.1) can be rewritten as

$$
\left\{\begin{array}{l}
\frac{d u}{d t}=b u\left(1-\frac{u}{c}\right)-u v \\
\frac{d v}{d t}=u v e^{-w}-v \\
\frac{d w}{d t}=a u v\left(1-e^{-w}+\rho\left(\frac{1-e^{-w}}{w}-1\right)\right)-d w
\end{array}\right.
$$

The equilibria of this scaled system are:

i) $\quad u=v=w=0$ (no empty patches; no herbivores; no predators)

ii) $\quad u=c, v=w=0$ (empty patches at carrying capacity; no herbivores; no predators)

iii) $u=1, v=b\left(1-c^{-1}\right), w=0$ (requires $c>1$; the density of empty patches is completely set by the "predation" pressure of the herbivores; no predators)

iv) $\quad v=b\left(1-\frac{u}{c}\right), w=\ln u,\left(1-\frac{u}{c}\right)\left(u-1+\rho \quad\left(\frac{u-1}{\ln u}-1\right)\right)=\frac{d}{a b} \ln u \quad$ (steady state with three trophic levels present; no explicit expression for $u$ )

The steady state (i) is unstable for all $b>0$, whereas (ii) is stable for $0<c<1$ and unstable for $c>1$. Linearization about the steady state (iii) yields the matrix

$$
\left[\begin{array}{ccc}
-\frac{b}{c} & -1 & 0 \\
b\left(1-\frac{1}{c}\right) & 0 & -b\left(1-\frac{1}{c}\right) \\
0 & 0 & a b\left(1-\frac{1}{c}\right)\left(1-\frac{1}{2} \rho\right)-d
\end{array}\right)
$$

which has eigenvalues

$$
\begin{aligned}
& \lambda_{1}=a b\left(1-\frac{1}{c}\right)\left(1-\frac{1}{2} \rho\right)-d \\
& \lambda_{2,3}=-\frac{b}{2 c} \pm \sqrt{\frac{b^{2}}{4 c^{2}}-b\left(1-\frac{1}{c}\right)} .
\end{aligned}
$$

Clearly $\operatorname{Re} \lambda_{2,3}<0$ for $c>1$, while $\lambda_{1}<0$ if and only if

$$
c\left(1-\frac{1}{2} \rho-\theta\right)<1-\frac{1}{2} \rho
$$

where by definition

$$
\theta=\frac{d}{a b} \text {. }
$$


The definition of $\rho$ (see 3.4) implies that $0 \leqslant \rho<1$ (we will make this hypothesis throughout the rest of the paper), so $1-\frac{1}{2} \rho>0$. Hence the steady state (iii) is stable for all values of $c>1$ if $1-\frac{1}{2} \rho-\theta<0$, whereas it is stable for $1<c<\frac{1-\frac{1}{2} \rho}{1-\frac{1}{2} \rho-\theta}$ and unstable for larger values of $c$ if $1-\frac{1}{2} \rho-\theta>0$.

We now turn our attention to the steady state (iv) which is only implicitly defined. The easiest way to proceed seems to change our point of view and consider $c$ as a function of $u$ :

$$
c(u)=\frac{u\left(u-1+\rho \frac{u-1-u \ln u}{\ln u}\right)}{u-1+\rho \frac{u-1-u \ln u}{\ln u}-\theta \ln u}
$$

We first investigate where and how this curve in the $(c, u)$-plane intersects the line $u=1$ corresponding to the steady state (iii). If we put $u=1+\epsilon$ and make a Taylor expansion with respect to $\epsilon$ we find

$c(1+\epsilon)=\frac{1-\frac{1}{2} \rho}{1-\frac{1}{2} \rho-\theta}+\epsilon \frac{\left(1-\frac{7}{12} \rho\right)\left(1-\frac{1}{2} \rho-\theta\right)-\left(1-\frac{1}{2} \rho\right)\left(\frac{1}{2} \theta-\frac{1}{12} \rho\right)}{\left(1-\frac{1}{2} \rho-\theta\right)^{2}}+$ h.o.t. (4.6)

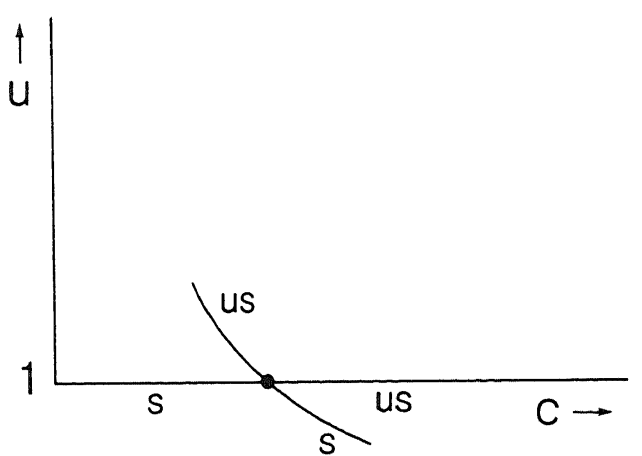

$q<0$

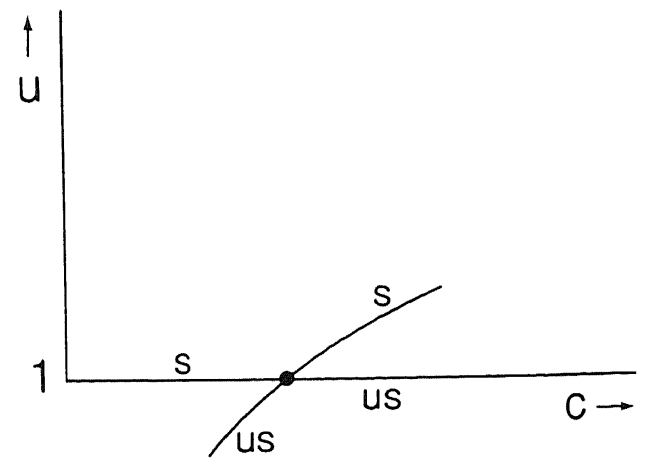

$q>0$

FIGURE 2

Local bifurcation diagram. $q$ is the coefficient of $\epsilon$ in (4.6). $s$ means stable and us unstable.

Thus we find, as to be expected, that for $1-\frac{1}{2} \rho-\theta<0$ no intersection occurs in the positive quadrant while for $1-\frac{1}{2} \rho-\theta>0$ intersection occurs exactly at the point where steady state (iii) loses its stability. Define $q=q(\rho, \theta)$ to be the coefficient of $\epsilon$ 
in the expansion then the local configuration is as depicted in Figure 2, where the stability assertions about steady state (iv) are based on the general principle of the exchange of stability in a bifurcation point (see Metz \& Diekmann (1986) VI. 1.2 and the references given there). Note that the branch with $u<1$ is biologically meaningless since $w<0$. The direction of bifurcation changes for $q=0$ which corresponds to $\theta=3(2-\rho)^{2} /(18-10 \rho)$ (see Figure 3).

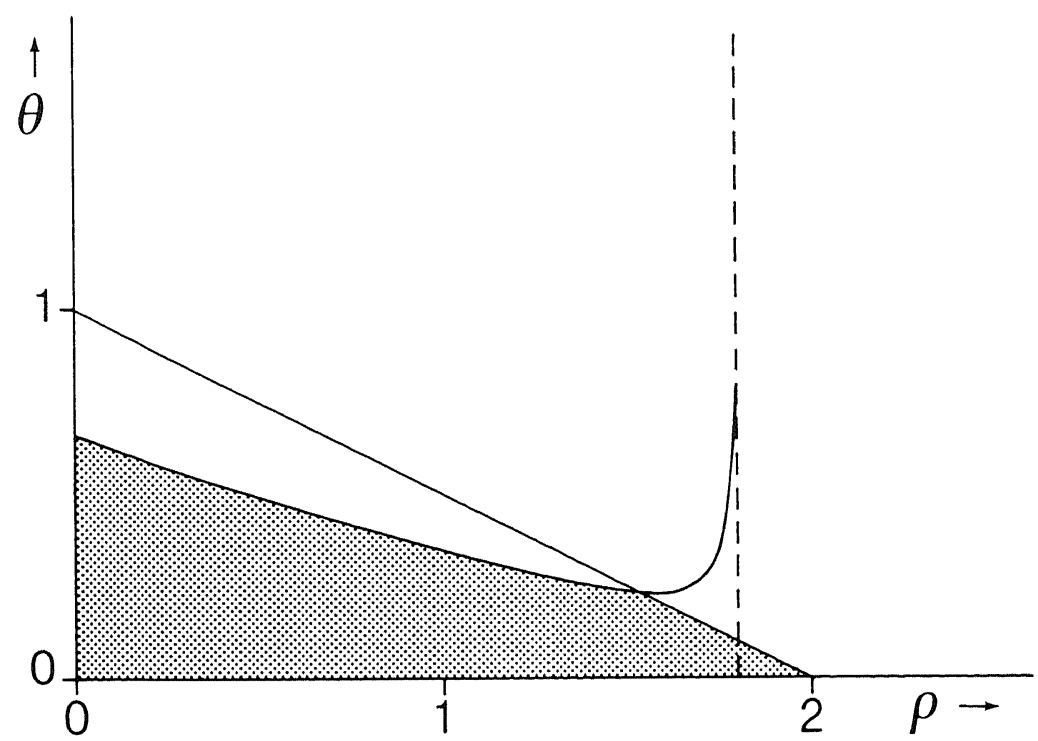

FIGURE 3

The set in the $(\rho, \theta)$-plane such that bifurcation occurs is the triangle $\rho, \theta \geqslant 0, \theta<1-\frac{1}{2} \rho$.

Within the hatched area bifurcation is supercritical and steady state (iv) is stable near the bifurcation point, whereas in the unhatched area of the triangle we have subcritical bifurcation and steady state (iv) is unstable near the bifurcation point.

Let us now try to obtain information about the global aspects of the bifurcation diagram. We have to address two partly related problems: what is the shape of $c(u)$ and what is the stability character. We begin by investigating the shape.

Since the numerator of $c(u)$ is always positive for $u>1$ and $0 \leqslant \rho<1$ the following lemma shows that $c(u)$ has precisely one positive branch for $u>1$.

LEMMA 4.1. The denominator of $c(u)$ as defined in (4.5) has no zero's for $u>1$ when $1-\frac{1}{2} \rho-\theta>0$ and precisely one zero when $1-\frac{1}{2} \rho-\theta<0$. 
Proof. We define

$$
\psi(z)=(1-\rho) z e^{z}+\rho e^{z}-\rho-z-\theta z^{2}
$$

and note that the zero's of $\psi$ with $z>0$ are in one to one correspondence with the zero's of the denominator via $u=e^{z}$. Then

$$
\begin{aligned}
& \psi^{\prime}(z)=(1-\rho) z e^{z}+e^{z}-1-2 \theta z \\
& \psi^{\prime \prime}(z)=(1-\rho) z e^{z}+(2-\rho) e^{z}-2 \theta \\
& \psi^{\prime \prime \prime}(z)=(1-\rho) z e^{z}+(3-2 \rho) e^{z}
\end{aligned}
$$

and consequently $\psi^{\prime \prime \prime}(z)>0$ for $z>0$. For $z \sim 0$ we have

$$
\psi(z) \sim\left(1-\frac{1}{2} \rho-\theta\right) z^{2}
$$

and for $z \rightarrow \infty$ we have $\psi(z) \rightarrow \infty$. Suppose $1-\frac{1}{2} \rho-\theta>0$. If $\psi$ has one positive zero it has to have at least two positive zero's. Since $\psi$ is increasing for small positive $z$ the function $\psi^{\prime}$ has to have at least two positive zero's as well. Consequently $\psi^{\prime \prime}$ has at least one positive zero. But $\psi^{\prime \prime}(0)=1-\frac{1}{2} \rho-\theta>0$ and $\psi^{\prime \prime \prime}(z)>0$ for $z>0$, so $\psi^{\prime \prime}$ cannot have a positive zero.

Next consider the case that $1-\frac{1}{2} \rho-\theta<0$. Then $\psi$ has an odd number of positive zero's. Assume this number is three or more. Since $\psi$ is decreasing for small positive $z$ the function $\psi^{\prime}$ has to have at least three positive zero's. Applying the same argument to $\psi^{\prime}$ we deduce that $\psi^{\prime \prime}$ has at least three positive zero's. However, since $\psi^{\prime \prime \prime}>0$ we know that $\psi^{\prime \prime}$ has only one positive zero. We conclude that $\psi$ cannot have more than one positive zero.

In principle the branch could have several wiggles and therefore we could have, for specific values of $c$, even more than two steady states with three trophic levels occupied. In the special case $\rho=0$ we can exclude the possibility of wiggles.

LEMMA 4.2. Let $c(u)$ be defined by (4.5). For $\rho=0$ and any $c>0$ the set $\{u>1 \mid c(u)=c\}$ contains at most two elements.

Proof. Define for fixed $c$ the function $F$ by

$$
F(u)=u(u-1)-c(u-1-\theta \ln u)
$$

Clearly there is a one to one correspondence between the zero's of $F$ with $u>1$ and the set $\{u>1 \mid c(u)=c\}$. Note that $F^{\prime \prime \prime}(u)=\frac{2 c \theta}{u^{3}}>0$ for $u>0$ and that $F(1)=0, F(\infty)=\infty$ and $F^{\prime}(1)=1-c(1-\theta)$. Employing the same arguments as in the proof of Lemma 4.1 it then follows that $F$ has precisely one zero for $u>1$ if $F^{\prime}(1)<0$ whereas $F$ has either no or two zero's for $u>1$ when $F^{\prime}(1)>0$. Note that $F^{\prime}(1)=0$ exactly at the bifurcation point $c=(1-\theta)^{-1}$. 


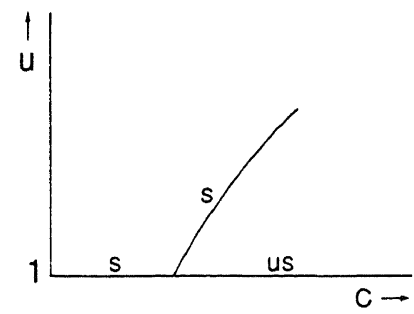

$1-\frac{1}{2} \rho-\theta>0, q>0$

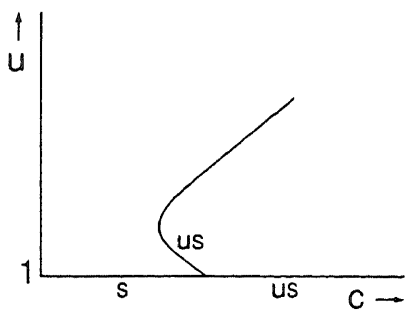

$1-\frac{1}{2} \rho-\theta>0, \mathrm{q}<0$

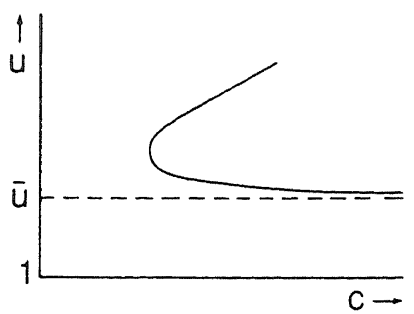

$1-\frac{1}{2} \rho-\theta<0$

FIGURE 4

Global bifurcation diagram (rigorously verified only for $\rho=0$ ). $s$ means stable and us unstable.

We have now verified the pictures of Figure 4 for the special case $\rho=0$. We conjecture that they are correct for $0<\rho<1$ as well, but for this we only have some numerical evidence obtained by solving $\frac{d}{d u} c(u)=0$ for $\theta$ and plotting $\theta$ so defined as a function of $u$, for many values of $\rho \in(0,1)$. The results suggest that for fixed $\rho$ and $\theta$ there is at most one turning point (i.e. a point where $\frac{d}{d u} c(u)=0$ ). We tried to exclude the "birth" of a turning point analytically by looking at second derivatives but even though one can reduce the problem to a quadratic equation in $\rho$ (with coefficients depending in a complicated way on $u$ ) we did not manage to find a proof.

Next we turn our attention to the stability problem. The Jacobi matrix at a steady state $c(u)=c$ is given by

$$
\left(\begin{array}{lcc}
-\frac{b}{c} u & -u & 0 \\
\frac{b}{u}\left(1-\frac{u}{c}\right) & 0 & -b\left(1-\frac{u}{c}\right) \\
\frac{d}{u} \ln u & \frac{d \ln u}{b\left(1-\frac{u}{c}\right)} & \frac{d \ln u g^{\prime}(\ln u)}{g(\ln u)}
\end{array}\right)
$$

where by definition

$$
g(w)=\frac{1-e^{-w}}{w}+\rho \frac{1-e^{-w}-w}{w^{2}} .
$$

The characteristic equation reads 


$$
\lambda^{3}+a_{1} \lambda^{2}+a_{2} \lambda+a_{3}=0
$$

where

$$
\begin{aligned}
& a_{1}=\frac{b}{c} u-d \ln u \frac{g^{\prime}(\ln u)}{g(\ln u)} \\
& a_{2}=-\frac{b d}{c} u \ln u \frac{g^{\prime}(\ln u)}{g(\ln u)}+d \ln u+b\left(1-\frac{u}{c}\right) \\
& a_{3}=b d\left(1-\frac{u}{c}\right) \ln u\left(\frac{u}{c-u}-1-\frac{g^{\prime}(\ln u)}{g(\ln u)}\right) .
\end{aligned}
$$

Note that $\lambda=0$ is a root iff $a_{3}=0$ and that $a_{3}=0$ iff either $u=1$ or $\frac{g^{\prime}(\ln u)}{g(\ln u)}=1-\frac{u}{c-u}$. The first possibility corresponds to the bifurcation from the $\left(u=1, v=b\left(1-\frac{1}{c}\right), w=0\right)$ branch and the second, as we are going to show, to the turning point(s) of the $c(u)$ branch. Indeed

$$
c(u)=\frac{u^{2} g(\ln u)}{u g(\ln u)-\theta}
$$

and therefore

$$
(u g(\ln u)-\theta)^{2} c^{\prime}(u)=\frac{u^{2} g^{2}(\ln u)}{c}\left\{(u-c) \frac{g^{\prime}(\ln u)}{g(\ln u)}+2 u-c\right\} .
$$

So $c^{\prime}(u)>0$ iff

$$
\frac{g^{\prime}(\ln u)}{g(\ln u)}<\frac{c-2 u}{u-c}=-1+\frac{u}{c-u} .
$$

It follows that for $u>1 a_{3}>0$ iff $c^{\prime}(u)>0$. In other words, at turning points of the $c(u)$ branch a real root changes from the left half plane (when $c^{\prime}(u)>0$; this follows from the Routh-Hurwitz criteria, see below) to the right half plane (when $\left.c^{\prime}(u)<0\right)$.

The stability of the steady state may also change by a pair of complex conjugated roots crossing the imaginary axis. Note that the characteristic equation has roots exactly on the imaginary axis iff $a_{2}>0$ and $a_{1} a_{2}=a_{3}$. Finally, recall that the Routh-Hurwitz criteria for stability are

$$
a_{1}>0, \quad a_{3}>0 \text { and } a_{1} a_{2}>a_{3}
$$

(and that this necessitates $a_{2}$ to be positive as well).

Armed with the above observations we will now show that the stability problem does not admit a simple solution. Clearly $c^{\prime}(u)<0$ and $u>1$ imply instability but the tempting conjecture that $c^{\prime}(u)>0$ and $u>1$ imply stability is false. A key point is that the $c(u)$ branch depends only on the compound parameter $\theta=\frac{d}{a b}$ whereas the coefficients $a_{1}, a_{2}$ and $a_{3}$ of the characteristic polynomial depend on $b$ and $d$ individually. If we let $a$ and $d$ tend to zero while keeping $b, \theta, \rho, u$ and $c$ constant the expressions for $a_{1}, a_{2}$ and $a_{3}$ show that both $a_{1}$ and $a_{2}$ are positive and bounded away from zero while $a_{3}$ tends to zero from above when $c^{\prime}(u)>0$. It follows that the Routh-Hurwitz stability criteria are satisfied for small $a$ and $d$. 
If, on the other hand, we let $a$ and $d$ tend to infinity while keeping all the other quantities constant then (note that $\left.g^{\prime}<0\right) a_{1}$ becomes negative and the steady state is unstable. In fact a pair of eigenvalues must cross the imaginary axis so that Hopf bifurcation theory implies the existence of a branch of periodic solutions of the system of ordinary differential equations.

We conclude that any given steady state on a part of the $c(u)$ branch with $c^{\prime}(u)>0$ may either be stable or unstable, depending on the precise values of the parameters $a, b$, and $d$. For small $a$ and $d$ and $1-\frac{1}{2} \rho-\theta<0$ or $1-\frac{1}{2} \rho-\theta>0, q<0$ we have at least two stable steady states. It seems very likely that for large $a$ and $d$ and $1-\frac{1}{2} \rho-\theta<0$ or $1-\frac{1}{2} \rho-\theta>0, q<0$ a stable steady state and a stable limit cycle coexist.

\section{BIOLOGICAL CONCLUSIONS}

The limiting ode system admits two stable steady states (as well as one unstable steady coexistence state) in a large domain of parameter space. In one of the stable steady states the predators are absent and the herbivores keep the number of "empty" host plant patches $n_{0}$ far below the carrying capacity $K$. If one tries to apply biological control by introducing a small number of predators the stability of this steady state prevents success. However, the introduction of a large number of predators may bring the system into the other stable steady-state in which the plants are almost at the carrying capacity (note that $c(u) \sim c$ for $u \rightarrow \infty$ ) while the herbivores are kept at a low level by the predators. There also exist regions in parameter space in which the latter steady state is unstable and, presumably, stable oscillations around this steady state exist.

In terms of the original parameters we have

$$
\theta=\frac{\nu x_{\max } \zeta}{\mu r \delta\left(x_{\max }-1\right)\left(\frac{\beta}{\gamma}+x_{\max }\right)}, \quad \rho=\frac{x_{\max }-1}{x_{\max }+\beta / \gamma} .
$$

Hence $\theta>1-\frac{1}{2} \rho$ if and only if

$$
x_{\max }<\frac{\nu \zeta}{\mu r \delta}-\frac{\beta}{\gamma}+\sqrt{\left(\frac{\nu \zeta}{\mu r \delta}-\frac{\beta}{\gamma}\right)^{2}+1+\frac{2 \beta}{\gamma}} .
$$

So if we think of situations with equal total exploitable host plant biomass $x_{\max } K$ then one can expect multiple stable steady states when there are many small patches and a single stable steady state when the patches are large but few.

6. REFERENCES

O. Diekmann, J.A.J. MeTz \& M.W. SABelis (1988). Mathematical models of predator-prey-plant interactions in a patchy environment, to appear in a special issue of Experimental and Applied Acarology edited by M.W. Sabelis, A.R.M. Janssen and W. Helle.

J.A.J. Metz \& O. Diekmann (eds.) (1986). The Dynamics of Physiologically Structured Populations. Springer Lecture Notes in Biomathematics 68. 